\title{
IMPACTO DE UM PROGRAMA PARA PROFILAXIA DE TROMBOEMBOLISMO VENOSO EM PACIENTES CLÍNICOS EM QUATRO HOSPITAIS DE SALVADOR
}

\author{
Ana Thereza C. Rocha ${ }^{*}$, Edison Ferreira de Paiva ${ }^{2}$, Danilo Miranda de Araújo ${ }^{3}$, Dulceane Natyara Cardoso ${ }^{3}$, Aline Cristina da Hora Pereira ${ }^{3}$, \\ Antônio Alberto Lopes ${ }^{4}$, Eduardo S. Darzé ${ }^{5}$ \\ Trabalho realizado no Hospital Universitário Professor Edgard Santos, Hospital Geral Roberto Santos, Hospital Português e Hospital Aliança, Salvador, BA
}

\section{*Correspondência:}

Rua Alberto Valença, 148 -

Apto. 203

Salvador - BA

CEP: $41810-825$

\begin{abstract}
RESUMO
Objetivo. Implementar um programa hospitalar de profilaxia de TEV através da criação de uma comissão, da realização de palestras e da distribuição de algoritmos baseados na Diretriz Brasileira para Profilaxia de TEV em Pacientes Clínicos e avaliar seu impacto na adequação da utilização de profilaxia em quatro hospitais de Salvador, Bahia.

Métodos. Foram realizados dois estudos de corte-transversal, um antes e um depois da implementação do programa, e comparadas as proporções de pacientes em risco de TEV e as mudanças na adequação da profilaxia.

Resultados. Foram avaliados 219 pacientes clínicos antes e 292 depois do programa. As taxas daqueles com pelo menos um fator de risco para TEV e daqueles com contra indicação $(\mathrm{Cl})$ para heparina foram semelhantes nos dois grupos: $95 \%$ vs. $98 \%(p=0,13)$ e $42 \%$ vs. $34 \%(p=0,08)$, respectivamente. Nos dois estudos, $75 \%$ vs. $82 \%(p=0,06)$ eram candidates para profilaxia, e $44 \%$ vs. $55 \%(p=0,02)$ eram candidatos sem qualquer $\mathrm{Cl}$ para heparina. Após o programa, utilizou-se mais profilaxia mecânica, $0,9 \%$ vs. $4,5 \%(p=0,03)$ e menos profilaxia farmacológica, $55,3 \%$ vs. $47,9 \%(p=0,04)$, embora tenha havido um aumento significativo na utilização das doses corretas das heparinas, $53 \%$ vs. $75 \%(p<0,001)$.

Conclusão. A profilaxia de TEV é subutilizada nos hospitais brasileiros. Aulas de educação continuada e distribuição passiva de algoritmos de profilaxia de TEV são insuficientes para melhorar a utilização, mas melhoram a adequação da profilaxia.
\end{abstract}

Unitermos: Tromboembolia venosa. Fatores de risco. Prevenção de doenças. Medicina interna. Guias de prática clínica como assunto. Heparina.

\section{INTRODUÇÃO}

Estudos controlados e randomizados em pacientes hospitalizados têm destacado que o risco de tromboembolismo venoso (TEV) em pacientes com condições clínicas diversas é comparável ao de pacientes cirúrgicos e que os eventos tromboembólicos podem ser efetivamente evitados com o uso de profilaxia. ${ }^{1-7}$ Estudos do tipo registro e enquetes em corte-transversal mostram que ainda existe extrema variabilidade na avaliação do risco e na utilização de profilaxia do TEV no Brasil e no mundo. ${ }^{8,9}$ Além disto, a prescrição de profilaxia frequentemente não segue as orientações dos consensos nacionais e internacionais. ${ }^{8,10} \mathrm{Em}$ estudo observacional, Arnold et al. ${ }^{11}$ demonstraram que 17,4\% dos casos de TEV eram potencialmente preveníveis e os motivos mais comuns para a falha na prevenção foram omissão da profilaxia (48\%), duração inadequada (23\%) e escolha incorreta do método profilático (21\%). Em estudo de corte-transversal em quatro hospitais públicos e privados de Salvador, foram avaliados 226 pacientes clínicos consecutivos e apenas um terço dos candidatos potenciais à prevenção do TEV recebiam profilaxia de forma adequada. ${ }^{12}$ Menna Barreto et al. demonstraram que em hospital universitário de Porto Alegre, entre 351 pacientes clínicos e cirúrgicos avaliados aleatoriamente, 96\% apresentavam fator de risco para TEV e contra indicações para heparina eram infrequentes (7\%), não justificando a não utilização de

1. Pneumologista, Intensivista, Doutora em Medicina e Saúde pela Universidade Federal da Bahia - UFBA; Mestre em Ciências da Saúde para Pesquisa Clínica pela Duke University; Pneumologista e coordenadora do Núcleo de Atenção e Tratamento do Tabagismo do Serviço de Pneumologia do Complexo Hospital Universitário Professor Edgard Santos e Professora Substituta do Departamento de Medicina da Universidade Federal da Bahia, Salvador, BA

2. Doutor em Emergências pela Universidade de São Paulo - USP; Professor Assistente do Serviço de Clínica Geral e Propedêutica do Hospital das Clínicas da Faculdade de Medicina da Universidade de São Paulo, São Paulo, SP

3. Médicos pela Faculdade de Medicina da Bahia - FMB da Universidade Federal da Bahia - UFBA, Salvador, BA

4. Doutor em Medicina e Saúde Pública; Professor Associado do Departamento de Medicina da Faculdade de Medicina da Bahia da Universidade Federal da Bahia UFBA, Salvador, BA

5. Mestre em Medicina e Saúde pela Universidade Federal da Bahia - UFBA e Chefe do Serviço de Cardiologia do Instituto Cardio Pulmonar, Salvador, BA 
profilaxia. ${ }^{13}$ Estes autores também mostraram que na unidade de terapia intensiva, na qual $79 \%$ dos pacientes criticamente enfermos foram classificados como risco moderado/alto de TEV, as medidas profiláticas foram prescritas para apenas $57 \% .{ }^{14}$ Em um registro brasileiro incluindo dados de mais de 27.000 pacientes cirúrgicos e clínicos, os autores mostraram que houve subutilização de profilaxia em $25 \%$ dos pacientes com alto risco e em $45 \%$ daqueles com risco moderado de TEV, sendo que a inadequação predominou entre os pacientes clínicos. ${ }^{15}$

Dificuldades com os métodos de avaliação de risco e várias outras barreiras pertinentes aos médicos e ao sistema de saúde fazem com que medidas efetivamente comprovadas e recomendadas em diretrizes demorem a ser aplicadas de maneira mais disseminada para benefício dos pacientes. Assim, o objetivo deste estudo foi o de avaliar o impacto da implementação de um programa de incentivo à profilaxia do TEV, baseado nas recomendações da Diretriz Brasileira para Profilaxia de TEV em Pacientes Clínicos, do Programa Diretrizes da Associação Médica Brasileira. ${ }^{16} \mathrm{~A}$ diretriz contém informações atuais e baseadas na evidência científica sobre a importância dos fatores de riscos individuais em pacientes clínicos, bem como recomendações sobre a eficácia dos métodos de profilaxia para TEV adaptadas a um algoritmo para avaliação de risco de TEV de fácil aplicabilidade. O programa inclui: 1) a distribuição e utilização destes algoritmos de avaliação de risco de TEV de forma rotineira; 2) a realização de palestras de educação continuada sobre a profilaxia do TEV para o corpo clínico do hospital e; 3) a formação de uma comissão hospitalar para a profilaxia do TEV para incentivar e coordenar estas medidas.

\section{Métodos}

\section{Desenho}

O estudo apresentava três etapas e foi realizado em quatro hospitais de Salvador, Bahia, que não tinham programas formais de incentivo à profilaxia contra TEV: Hospital Português, Hospital Aliança, Hospital Universitário Professor Edgard Santos e Hospital Geral Roberto Santos. Na primeira etapa, em março de 2005, foi realizado nos quatro hospitais um estudo de cortetransversal durante um dia, avaliando a utilização e adequação da profilaxia de TEV nos pacientes clínicos internados, antes da implementação do programa.

Na segunda etapa, a partir de 2007 e durante um período de doze meses, foi paulatinamente implementado um programa de profilaxia de TEV baseado nas recomendações da Diretriz Brasileira de Profilaxia de TEV em Pacientes Clínicos Internados que constava de vários passos. Os primeiros eram a identificação de profissionais interessados na causa do TEV e o engajamento da diretoria do hospital. A partir daí, para que o programa fosse amplamente aceito e seguido pelo corpo clínico do hospital, era fundamental que fosse criada uma Comissão de Profilaxia de TEV (CPTEV). Era recomendado que a CPTEV fosse multidisciplinar, composta por pelo menos um médico clínico, um cirurgião e um membro da enfermagem e que atuasse de modo semelhante às Comissões de Controle de Infecção Hospitalar, com atitude pró-ativa e respaldada pela diretoria do hospital. Havendo disponibilidade e, dependendo das características do hospital, recomendava-se também a participação de membros da farmácia clínica, da fisioterapia e de profissionais ligados ao controle de qualidade hospitalar. Entre as atividades da CPTEV, devia constar a avaliação programada da utilização de profilaxia do TEV. Para isto, a CPTEV deveria viabilizar junto às instâncias necessárias (ex. comissão de prontuários, comissão de farmácia e terapêutica e informática) as adaptações necessárias dos algoritmos de avaliação de risco, facilitando a sua disponibilização nas enfermarias e nos prontuários médicos. Além disto, a CPTEV deveria promover e divulgar palestras padronizadas de educação continuada, ministradas por especialistas na área de TEV para os membros do corpo clínico do hospital, incluindo a enfermagem. Em uma delas, eram apresentados dados epidemiológicos sobre a profilaxia em pacientes clínicos no Brasil e no mundo, e era realizada discussão de casos clínicos que abordavam a utilização dos algoritmos de avaliação de risco e de profilaxia de TEV. Em outra, abordavam-se as diretrizes nos cuidados a causas frequentes de internação clínica (insuficiência cardíaca congestiva, exacerbação aguda de doença pulmonar obstrutiva crônica e pneumonia), incluindo a profilaxia de TEV. Uma terceira palestra era voltada especificamente para a enfermagem e, além de identificação do risco e prescrição de profilaxia, eram discutidos aspectos específicos como a avaliação diária do paciente e os cuidados na administração das medicações profiláticas. Além das palestras, eram distribuídos materiais educativos como algoritmos de avaliação de risco, etiquetas de alerta, brochuras sobre o programa e pôsteres plastificados reproduzindo os algoritmos para fixação nas salas de prescrição.

Na última etapa, em março de 2008, foi realizado outro estudo de corte-transversal durante um único dia, avaliando o impacto do programa na utilização e na adequação da profilaxia de TEV entre os pacientes clínicos internados.

Os comitês institucionais locais de ética em pesquisa concederam aprovação para realização de todas as fases do estudo e um termo de consentimento livre e esclarecido foi obtido de todos os pacientes.

Foram coletados dados sobre características de cada hospital (tipo de hospital, público ou privado e presença de programa de residência médica), características demográficas e biométricas (idade, sexo, raça, peso e altura) dos pacientes, diagnóstico principal durante a admissão e histórico detalhado sobre fatores de risco (FR) para TEV: história prévia de TEV, câncer, quimioterapia, hormonioterapia, gravidez, puerpério, trombofilia hereditária ou adquirida, terapia de reposição hormonal, cateteres venosos centrais, insuficiência cardíaca congestiva (ICC), infarto agudo do miocárdio (IAM), insuficiência vascular periférica, varizes, paresia/paralisia de membros inferiores, acidente vascular cerebral isquêmico (AVCI), acidente vascular cerebral hemorrágico (AVCH), insuficiência respiratória com e sem ventilação mecânica, doença pulmonar obstrutiva crônica (DPOC), pneumonia, doenças inflamatórias intestinais, doenças reumáticas ativas, infecções e síndrome nefrótica. Foram coletados também dados sobre: data de início e interrupção e posologia da profilaxia farmacológica com heparina não fracionada (HNF), heparina de baixo peso molecular (HBPM) ou warfarin e contra indicações gerais para heparina: insuficiência renal com clearance de creatinina calculado inferior a $30 \mathrm{ml} / \mathrm{min}$; alergia à heparina; sangramento ativo, definido como sangramento macroscópico de qualquer local, excluindo locais de punção venosa; coagulopatia, definida como plaquetas $<100.000 / \mathrm{mm}^{3}$, tempo de tromboplastina 
parcial ativada (TTPA) $>60$ segundos ou razão normalizada internacional $(\mathrm{RNI})>1,5$, sem uso de warfarin, ou cirurgia ocular ou cerebral há menos de duas semanas. A prescrição de métodos físicos de profilaxia com meias elásticas de compressão gradual (MECG), ou compressão pneumática intermitente (CPI) e a prescrição de fisioterapia motora também foram registradas.

Eram considerados "candidatos à profilaxia" os pacientes com mobilidade reduzida e idade $\geq 40$ anos, com pelo menos um FR para TEV, ou, se idade $<40$ anos, aqueles com pelo menos dois FR para TEV. Mobilidade reduzida foi definida como repouso no leito devido à doença, por pelo menos metade do dia, excluindo o período do sono ou a presença de imobilidade crônica. "Candidatos à profilaxia sem contra indicações", eram os pacientes "candidatos à profilaxia" sem qualquer contra indicação ao uso de heparina. Foi considerado "uso de qualquer profilaxia" a prescrição, em qualquer momento da hospitalização, de pelo menos um método de profilaxia farmacológica ou mecânica. A avaliação da "adequação da profilaxia" foi considerada para os "candidatos à profilaxia" que receberam profilaxia farmacológica e baseou-se nas recomendações da Diretriz Brasileira para Profilaxia de TEV em Pacientes Clínicos, ou seja, HNF 5.000 UI de 8-8 h, ou a dose recomendada para cada HBPM: enoxaparina $40 \mathrm{mg} /$ dia, dalteparina $5.000 \mathrm{UI} /$ dia ou nadroparina de acordo com o peso do paciente.

Caracterizava-se subutilização de profilaxia quando o paciente era candidato, mas não recebia qualquer profilaxia; superutilização, quando não era candidato e recebia profilaxia; e utilização adequada se o paciente era candidato e recebia profilaxia farmacológica, ou se era candidato e recebia profilaxia mecânica por apresentar risco de sangramento.

Análise estatística:

Os dados são apresentados como proporções, médias ( \pm desvios padrão) e medianas ( \pm distâncias interquartílicas), quando apropriado para os estudos antes e depois. As variáveis contínuas foram comparadas pelo teste $T$ e Student, quando a distribuição era normal. A idade dos pacientes foi tratada como variável contínua e como categórica, utilizando 40 e 55 anos como pontos de corte. As proporções de utilização e adequação dos métodos de profilaxia entre os estudos antes e depois foram comparados com os testes de Qui quadrado ou Fisher, conforme apropriado. Os valores de $p<0,05$ estabeleceram o nível de significância estatística. As análises foram feitas com o software SPSS 9.0 for Windows.

\section{Resultados}

A apresentação do programa de profilaxia de TEV à diretoria clínica foi feita pelos investigadores principais, com o intuito de promover a criação da CPTEV. A distribuição dos algoritmos para avaliação de risco e a realização das palestras do programa de educação continuada ocorreram de acordo com as possibilidades oferecidas pela direção e chefias específicas das enfermarias do hospital. A aceitação e a efetivação das iniciativas apresentaram grande variação entre os hospitais participantes e mesmo entre as enfermarias em cada instituição. Inúmeras barreiras ao processo de implementação foram identificadas e, de um modo geral, foram peculiares ao tipo de hospital, público ou privado, e à presença de residência médica. Os principais obstáculos encontrados durante a fase de implementação foram: a mudança da diretoria clínica nos quatro hospitais estudados, a saída do investigador principal de dois dos hospitais e mudanças no corpo clínico ou no grupo de residentes, o que prejudicou a continuidade do processo.

As palestras do programa foram ministradas, mas o intervalo que foi possível entre estas e a segunda coleta de dados superou em muito a previsão do estudo, estendendo-se por mais de um ano. Três dos hospitais atingiram a meta de três palestras, enquanto em um dos hospitais privados, por questões administrativas internas, apenas uma palestra foi ministrada. Neste mesmo hospital, a distribuição dos algoritmos de avaliação de risco ficou restrita à unidade de terapia intensiva, enquanto que nos demais hospitais a distribuição foi feita em todas as enfermarias clínicas.

No primeiro estudo, foram incluídos 226 e no segundo 298 pacientes clínicos. Treze pacientes (sete antes e seis depois) foram excluídos das análises por apresentarem o diagnóstico de TEV na admissão hospitalar, sendo analisados 219 e 292 pacientes, respectivamente. As características gerais e os diagnósticos de admissão dos pacientes, no primeiro e no segundo estudo, são mostradas na Tabela 1 . O percentual de mulheres foi de $52 \%$ vs. $51 \%$ e as médias de idades dos pacientes foram 58 vs. 55 anos, nos estudos antes e depois do programa. Quanto ao tipo de hospital, houve um número significativamente maior de pacientes em hospitais públicos no estudo depois: $51,1 \%$ vs. $60,3 \%, p=0,05$.

Os FR para TEV são mostradas na Tabela 2 e as contra indicações relativas e absolutas para profilaxia farmacológica na Tabela 3. A média de FR por paciente foi de 2,6 vs. 3,2 e a média de contra indicações de 0,47 vs. 0,46 nos estudos antes e depois do programa. Os percentuais de pacientes com pelo menos um FR foram de $94 \%$ vs. $97 \%$ e com pelo menos uma contra indicação de $42 \%$ vs. 34\%, respectivamente. A mobilidade reduzida foi descrita em $79 \%$ dos pacientes no primeiro estudo vs. $84 \%$ no segundo. Antes da implementação do programa, $75,3 \%$ (165/219) eram candidatos a profilaxia vs. $82,2 \%(240 / 292)$ após o programa, enquanto 44,3\% (97/219) vs. $55,1 \%$ (161/292) eram candidatos e não apresentavam qualquer contra indicação à heparina (Tabela 3).

Quanto à utilização de alguma forma de profilaxia, houve menor uso da profilaxia farmacológica, 55,3\% (121/219) vs. $45,9 \%$ (134/292), $p=0,04$, mas houve um aumento no uso de profilaxia mecânica no segundo estudo, 0,9\% (2/219) vs. 4,5\% (13/292), $p=0,03$ (Tabela 4). A utilização de profilaxia não diferiu entre os hospitais públicos e privados, nos estudos antes e depois: $52,7 \%$ vs. $57,9 \%$ ( $p=N S$ ) e $44,3 \%$ vs. $48,3 \%$ $(p=N S)$, respectivamente, mas foi significativamente menor entre os dois estudos em apenas um hospital público, 51,9\% vs. $31,5 \%(p=0,03)$.

Entre os candidatos à profilaxia, não houve diferenças significativas entre os dois estudos quanto à adequação do uso de profilaxia farmacológica ou profilaxia mecânica (se o paciente tinha contra indicação à heparina), 60,6\% (100/165) vs. $51,3 \%$ (123/240) ou subutilização, 39,4\% (65/165) vs. 48,8\% $(117 / 240), p=0,07$. Entre os não candidatos à profilaxia, houve menor superutilização de profilaxia no segundo estudo, 40,7\% (18/52) vs. 34,6\% (22/54), mas não de forma significativa, $p=0,55$. A adequação de uso entre os candidatos à profilaxia foi 
Tabela 1 - Características gerais dos pacientes clínicos nos estudos antes e depois do programa de educação continuada

\begin{tabular}{|c|c|c|c|}
\hline Variáveis & $\begin{array}{c}\text { Antes } \\
(\mathrm{N}=219)\end{array}$ & $\begin{array}{c}\text { Depois } \\
(\mathrm{N}=292)\end{array}$ & $\mathbf{P}$ \\
\hline $\begin{array}{l}\text { Idade, média } \pm \text { DP } \\
\qquad 40 \text { anos, \% (N) } \\
>55 \text { anos, \% (N) }\end{array}$ & $\begin{array}{c}57,9 \pm 19,3 \\
79,0(173) \\
58,9(129)\end{array}$ & $\begin{array}{c}54,9 \pm 19,4 \\
77,7(227) \\
51,7(150)\end{array}$ & $\begin{array}{l}\text { NS } \\
\text { NS } \\
0,13\end{array}$ \\
\hline Gênero masculino, \% (N) & $48,4(106)$ & $48,6(150)$ & NS \\
\hline Peso, média $\pm \mathrm{DP}$ & $61,7 \pm 12,7$ & $62,3 \pm 12,1$ & NS \\
\hline $\begin{array}{l}\text { Creatinina, média } \pm \mathrm{DP} \\
\quad \text { Mediana } \pm \mathrm{DIQ}\end{array}$ & $\begin{array}{l}\text { ND } \\
\text { ND }\end{array}$ & $\begin{array}{l}1,9 \pm 3,0 \\
0,9 \pm 0,7\end{array}$ & - \\
\hline Clearance de creatinina, média \pm DP & ND & $68,8 \pm 54,0$ & - \\
\hline $\begin{array}{l}\text { Tempo de internação em dias, média } \pm \text { DP } \\
\quad \text { Mediana } \pm \text { DIQ }\end{array}$ & $\begin{aligned} 23,4 & \pm 33,4 \\
14 & \pm 25\end{aligned}$ & $\begin{aligned} 23,4 & \pm 39,2 \\
12 & \pm 31\end{aligned}$ & NS \\
\hline $\begin{array}{l}\text { Diagnóstico principal de admissão } \\
\text { ICC } \\
\text { IAM } \\
\text { Cardíaco outro } \\
\text { Pneumonia } \\
\text { Outras doenças respiratórias (ex. DPOC) } \\
\text { AVCI } \\
\text { AVCH } \\
\text { Neurológicos outros } \\
\text { Sangramento gastrintestinal } \\
\text { Doença inflamatória intestinal } \\
\text { Outras doenças gastrintestinais } \\
\text { Problema do acesso renal para HD } \\
\text { Doenças renais outras } \\
\text { Infecção localizada } \\
\text { Infecção sistêmica } \\
\text { Câncer hematológico } \\
\text { Câncer de órgão sólido } \\
\text { Doença reumática ativa } \\
\text { Doença com risco de hemorragia } \\
\text { Outro }\end{array}$ & $\begin{array}{c}5,5(12) \\
3,7(8) \\
5,0(11) \\
7,8(17) \\
5,0(11) \\
7,3(16) \\
3,7(8) \\
4,1(9) \\
0,9(2) \\
2,7(6) \\
9,6(21) \\
0,5(1) \\
13,7(30) \\
3,7(8) \\
3,2(7) \\
5,0(11) \\
6,4(14) \\
2,7(6) \\
0,5(1) \\
9,1(20)\end{array}$ & $\begin{array}{c}6,8(20) \\
1,0(3) \\
4,5(13) \\
7,9(23) \\
3,4(10) \\
4,1(12) \\
1,0(3) \\
8,2(24) \\
2,1(6) \\
0,3(1) \\
9,2(27) \\
1,4(4) \\
6,8(20) \\
11,0(32) \\
6,2(18) \\
5,1(15) \\
11,3(33) \\
0,7(2) \\
1,0(3) \\
7,9(23)\end{array}$ & \\
\hline $\begin{array}{l}\text { Tipo de hospital } \\
\text { Público } \\
\text { Privado }\end{array}$ & $\begin{array}{l}51,1(112) \\
48,9(107)\end{array}$ & $\begin{array}{l}60,3(176) \\
39,7(116)\end{array}$ & 0,047 \\
\hline Hospital com programa de residência médica & $51,1(112)$ & $60,3(176)$ & 0,047 \\
\hline
\end{tabular}

Notas: DP indica desvio padrão; DIQ, distância interquartílica; IMC, índice de massa corpórea; ND, não disponível; ICC, insuficiência cardíaca congestiva; IAM, infarto agudo do miocárdio; DPOC, doença Notas: DP indica desvio padrão; DIQ, distância interquartilica; IMC, indice de massa corporea; $\mathrm{ND}$, não disponivel; ICC, insuficiência cardiaca
pulmonar obstrutiva crônica; $\mathrm{AVCl}$, acidente vascular cerebral isquêmico; $\mathrm{AVCH}$, acidente vascular cerebral hemorrágico e $\mathrm{HD}$, hemodiálise. 


\begin{tabular}{|c|c|c|c|}
\hline \multicolumn{4}{|c|}{$\begin{array}{c}\text { Tabela } 2 \text { - Fatores de risco para TEV em pacientes clínicos antes } \\
\text { e depois do programa de educação continuada }\end{array}$} \\
\hline Variáveis & $\begin{array}{c}\text { Antes } \\
(\mathrm{N}=219)\end{array}$ & $\begin{array}{c}\text { Depois } \\
(\mathrm{N}=292)\end{array}$ & $P$ \\
\hline Redução da mobilidade \% (N) & 78,5 (172) & $83,4(244)$ & 0,17 \\
\hline FR para TEV, \% (N) & 95,4 (209) & $97,9(286)$ & 0,13 \\
\hline História prévia de TEV & $4,2(18)$ & $2,4(7)$ & 0,033 \\
\hline História familiar de TEV & $4,6(10)$ & $3,4(10)$ & 0,65 \\
\hline Câncer hematológico & $5,9(13)$ & $6,8(20)$ & 0,72 \\
\hline Câncer de órgão sólido & $10,0(22)$ & $15,8(46)$ & 0,06 \\
\hline Quimioterapia ou hormonioterapia & $8,7(19)$ & $12,3(36)$ & 0,20 \\
\hline Trombofilia & 0 & $1,4(4)$ & 0,14 \\
\hline ICC & $4,6(10)$ & $11,6(34)$ & 0,006 \\
\hline IAM & $3,7(8)$ & $5,1(15)$ & 0,52 \\
\hline Varizes e insuf. vascular & $21,5(47)$ & $30,5(89)$ & 0,03 \\
\hline Síndrome nefrótica & $2,3(5)$ & $3,4(10)$ & 0,60 \\
\hline Gravidez e puerpério & $0,9(2)$ & $1,4(4)$ & 0,70 \\
\hline Abortamento repetido & $10,0(22)$ & $2,1(6)$ & 0,0001 \\
\hline Anticoncepcional oral & $1,8(4)$ & $2,7(8)$ & 0,57 \\
\hline Terapia de reposição hormonal & 0 & $0,3(1)$ & 1,0 \\
\hline $\mathrm{AVCl}$ & $14,1(33)$ & $8,6(25)$ & 0,02 \\
\hline $\mathrm{AVCH}$ & $5,9(13)$ & $1,7(5)$ & 0,01 \\
\hline Paresia ou paralisia de membros inferiores & $30,1(66)$ & $26,0(76)$ & 0,32 \\
\hline Infecção localizada ou sistêmica & $38,8(85)$ & $51,4(150)$ & 0,005 \\
\hline Doenças respiratórias graves & $10,5(23)$ & $23,3(68)$ & 0,0001 \\
\hline Doença inflamatória intestinal & $2,7(6)$ & $0,3(1)$ & 0,05 \\
\hline Doença reumática ativa & $5,9(13)$ & $3,1(9)$ & 0,13 \\
\hline Obesidade & $10,5(23)$ & $4,5(13)$ & 0,01 \\
\hline CVC & $29,2(64)$ & $41,8(122)$ & 0,004 \\
\hline CVC para HD & $11,4(25)$ & $15,4(45)$ & 0,24 \\
\hline UTI & $16,0(35)$ & $27,1(79)$ & 0,004 \\
\hline Ventilação mecânica & $7,8(17)$ & $24,3(71)$ & 0,0001 \\
\hline
\end{tabular}

Notas: FR indica fatores de risco; TEV, tromboembolismo venoso; ICC, insuficiência cardíaca congestiva; IAM, infarto agudo do miocárdio; $\mathrm{AVCl}$, acidente vascular cerebral isquêmico; $\mathrm{AVCH}$ acidente vascular cerebral hemorrágico; CVC, cateter venoso central; HD, hemodiálise e UTI, unidade de terapia intensiva.

\begin{tabular}{|c|c|c|c|}
\hline Variáveis & $\begin{array}{c}\text { Antes } \\
(\mathrm{N}=219)\end{array}$ & $\begin{array}{c}\text { Depois } \\
(\mathrm{N}=292)\end{array}$ & $\mathbf{P}$ \\
\hline $\begin{array}{l}\text { Candidatos à profilaxia de TEV, \% } \\
\text { (N) }\end{array}$ & 75,3 (165) & $82,2(240)$ & 0,06 \\
\hline $\begin{array}{l}\text { Contra indicações, \% (N) } \\
\text { Disfunção renal } \\
\text { Insuficiência renal moderada } \\
\text { Hemorragia intracraniana } \\
\text { Plaquetas (<100.000/mm³) } \\
\text { Sangramento ao ser internado } \\
\text { Disfunção hepática } \\
\text { Úlcera gastroduodenal ativa } \\
\text { Alergia a heparina }\end{array}$ & $\begin{array}{c}42,0(92) \\
6,8(15) \\
16,4(36) \\
4,6(10) \\
4,6(10) \\
5,5(11) \\
7,3(16) \\
2,3(5) \\
0\end{array}$ & $\begin{array}{c}34,2(100) \\
8,2(24) \\
13,4(39) \\
1,4(4) \\
7,9(23) \\
6,2(18) \\
4,5(13) \\
0,7(2) \\
0,7(2)\end{array}$ & $\begin{array}{l}0,08 \\
0,61 \\
0,40 \\
0,05 \\
0,15 \\
0,70 \\
0,18 \\
0,14 \\
0,50\end{array}$ \\
\hline $\begin{array}{l}\text { Candidatos à profilaxia sem contra } \\
\text { indicações, \% (N) }\end{array}$ & 44,3 (97) & $55,1(161)$ & 0,02 \\
\hline
\end{tabular}

Nota: TEV indica tromboembolismo venoso.
Tabela 4 - Utilização e adequação de profilaxia de TEV para todos os pacientes antes e depois do programa de educação continuada.

\begin{tabular}{lccc}
\hline Variáveis & Antes & Depois & P \\
\hline Utilização da profilaxia & & & \\
Profilaxia não utilizada & $44,7(98)$ & $54,1(158)$ & 0,04 \\
Profilaxia farmacológica & $55,3(121)$ & $45,9(134)$ & 0,04 \\
Combinação de & $0,5(1)$ & $2,1(6)$ & - \\
farmacológica e mecânica & $0,9(2)$ & $4,5(13)$ & 0,03 \\
Profilaxia mecânica & $0,5(1)$ & $2,4(7)$ & - \\
Profilaxia mecânica isolada & $\mathrm{ND}$ & $50,3(147)$ & - \\
Fisioterapia & & & \\
Adequação da profilaxia* & & & \\
Subutilização & $29,7(65)$ & $40,1(117)$ & \\
Superutilização & $10,0(22)$ & $6,2(18)$ & \\
Utilização adequada & $45,7(100)$ & $42,1(123)$ & \\
Não indicada & $14,6(32)$ & $11,6(34)$ & \\
\hline
\end{tabular}

Notas: TEV indica tromboembolismo venoso. *Subutilização: paciente era candidato, mas não recebeu qualquer profilaxia; superutilização: paciente não era candidato e recebeu profilaxia; utilização adequada: paciente era candidato e recebeu profilaxia farmacológica ou profilaxia mecânica se havia risco de sangramento.

Tabela 5 - Escolha de métodos de profilaxia de TEV para pacientes antes e depois do programa de educação continuada.

\begin{tabular}{lclc}
\hline Variáveis & Antes & Depois & $\mathbf{P}$ \\
\hline Escolha inicial de profilaxia & & & 0,62 \\
HBPM & $55,4(67)$ & $47,8(64)$ & \\
HNF & $43,0(52)$ & $50,7(68)$ & \\
Warfarin & $1,7(2)$ & $1,5(2)$ &
\end{tabular}

Doses de anticoagulantes utilizados

0,0001

HBPM

$\begin{array}{lcc}\text { Enoxaparina } 40 \text { mg/dia } & 46,3(56) & 36,6(49) \\ \text { Outras doses de enoxaparina } & 8,3(10) & 6,0(8) \\ \text { HNF } & & \\ 5.000 \text { UI 8-8 h } & 6,6(8) & 37,3(50) \\ 5.000 \text { UI 12-12 h } & 38,8(47) & 20,1(27)\end{array}$

Adequação das doses

Enoxaparina $40 \mathrm{mg} / \mathrm{dia}$ ou HNF

$\begin{array}{lcc}5.000 \mathrm{UI} 8-8 \mathrm{~h} & 52,9(64) & 74,6(100) \\ \text { HNF 5.000 UI 12-12 h } & 38,8(47) & 19,4(26) \\ \text { Outras doses sistêmicas } & 8,3(10) & 6,0(8)\end{array}$

0,001

Notas: TEV indica tromboembolismo venoso; HBPM, heparina de baixo peso molecular e HNF, heparina não fracionada.

maior em hospitais privados que públicos nas duas fases, mas também não de forma significativa: $68,5 \%$ vs. $62,1 \%(p=0,44)$ e $51,6 \%$ vs. $59,0(p=0,25)$.

Quando os 255 pacientes que receberam profilaxia farmacológica (121 no primeiro estudos e 134 no segundo) foram avaliados em relação às recomendações da diretriz para uso de doses profiláticas altas, houve uma aumento significativo no segundo estudo do uso correto de enoxaparina (40 mg/dia) e HNF (5.000 UI 8-8h), de 52,9\% para 74,6\%, p<0,001 e uma concomitante diminuição do uso da dose profilática baixa de HNF (5.000 UI 12-12 h), de 38,8\% para 19,4\%, p<0,001 (Tabela 5). 


\section{Discussão}

Neste estudo foram avaliados o risco de TEV entre pacientes clínicos internados e a utilização e adequação da sua profilaxia em dois momentos distintos, antes e após a implementação de medidas de educação continuada nos hospitais participantes. O estudo propôs a criação de uma comissão em cada hospital para incentivar a profilaxia do TEV através de palestras educativas sobre a profilaxia do TEV e da incorporação de algoritmos de avaliação do risco de TEV aos prontuários e à rotina hospitalar. Inúmeras dificuldades foram encontradas neste processo em cada um dos hospitais participantes, desde desconfiança de membros da direção quanto à real necessidade e à ética das recomendações, até problemas burocráticos para a adaptação dos protocolos e dificuldades para a realização das palestras propostas pelo programa. Quanto ao corpo clínico, as dúvidas mais frequentes foram relativas à necessidade do uso dos algoritmos para a avaliação do risco e à segurança e disponibilidade dos métodos de profilaxia na instituição e após a alta hospitalar. Algumas barreiras citadas para o uso rotineiro dos algoritmos foram a falta de tempo para a avaliação e prescrição de profilaxia e a falta de uma política hospitalar incentivando a proposta do programa. Além disto, a falta de um funcionário específico para a comissão e a percepção de aumento do trabalho e do custo com o uso de profilaxia de TEV, por parte da administração dos hospitais, revelou-se outra barreira importante ao programa.

No Brasil já existem alguns estudos que atestam a subutilização de profilaxia de TEV ${ }^{12,13,15}$, no entanto propostas práticas de como modificar esta realidade são escassas. Um estudo randomizado em hospital americano demonstrou que gerar alertas automáticos para os médicos caso os seus pacientes tenham fatores de risco para TEV (de acordo com dados clínicos), inquerindo sobre a utilização de profilaxia, resultou não só na melhora significativa da utilização de profilaxia, como também na redução de TEV clinicamente aparente ${ }^{17}$. Em um hospital universitário de Belo Horizonte, Sad et al. realizaram um estudo, em dois momentos, sobre a implementação de uma comissão de profilaxia de TEV com respaldo completo da diretoria ${ }^{18}$. Foram aplicadas medidas educativas para o corpo clínico (palestras e impressos) e auditorias semanais de um percentual de pacientes internados por enfermeira da comissão, dando retorno aos médicos quanto ao seu desempenho. Com estas estratégias, os autores evidenciaram um aumento significativo no percentual de adequação de profilaxia entre pacientes clínicos ( $62 \%$ vs. $82 \%$, $p<0,05)$ e cirúrgicos ( $49 \%$ vs. $75 \%, p<0,05$ ).

Uma limitação deste estudo é que, devido às barreiras encontradas, a fase de implementação das medidas, seguida da nova coleta de dados, teve de ser estendida por mais de um ano, alterando o desenho inicial do estudo. Outra limitação refere-se à avaliação pontual e não prospectiva do uso de profilaxia. 0 desenho de corte-transversal foi escolhido pela praticidade da coleta de dados com potencial para estimar a situação da prática de profilaxia, embora compreenda-se que, com este tipo de avaliação, pode ter havido sub ou superestimação do uso correto de profilaxia, principalmente em relação à duração mínima recomendada.

Quanto ao risco de TEV, este estudo reforça o fato de que os FR são extremamente frequentes em pacientes clínicos internados, uma vez que mais de $90 \%$ deles apresentavam pelo menos um FR, em ambos os estudos. Uma taxa semelhante foi apresentada no estudo IMPROVE, um registro prospectivo com mais de 12.515 pacientes sobre a utilização de profilaxia em pacientes clínicos de 11 países, incluindo 605 no Brasil. ${ }^{9}$ As contra indicações para profilaxia foram comuns, mas não deveriam inviabilizar o uso de métodos mecânicos de profilaxia, como as meias elásticas de compressão e dispositivos de compressão pneumática das pernas. Neste sentido, houve um aumento significativo no uso de métodos mecânicos no estudo depois do programa ( $4,5 \%$ vs. $0,9 \%, p=0,03$ ). Apesar de inexistirem estudos clínicos demonstrando a efetividade de fisioterapia motora, registrou-se prescrição da fisioterapia em $50,3 \%$ dos pacientes no segundo estudo. Considerando que a prescrição de fisioterapia motora já faz parte da cultura médica, a participação dos fisioterapeutas na avaliação regular do risco de TEV, baseando-se no nível de mobilidade dos pacientes e na presença de FR, deveria ser encarada como uma oportunidade para a melhorar o uso da profilaxia do TEV.

A utilização e a adequação de profilaxia farmacológica foram menores no segundo estudo, ao passo que a subutilização aumentou. Apenas a superutilização de profilaxia, para aqueles que não eram candidatos, foi menor na segunda fase. Isto ocorreu apesar de a avaliação rotineira do risco de TEV e do uso de doses profiláticas efetivas e seguras para pacientes clínicos ${ }^{19}$ serem claramente mostrados nos algoritmos de avaliação de risco disponibilizados pelo programa e reforçados em todas as palestras padronizadas. Em uma metanálise recente ${ }^{20}$, foi demonstrado que as doses de HNF 5.000 UI de 8-8 h são significativamente mais efetivas do que as doses de HNF 5.000 UI de 12-12 h. Consequentemente, dois achados importantes foram o significativo aumento no segundo estudo do uso correto das doses profiláticas altas preconizadas pela Diretriz Brasileira, tanto para enoxaparina quanto para HNF, passando de $52,9 \%$ para $74,6 \%$, e uma concomitante diminuição do uso da dose profilática baixa de HNF de $38,8 \%$ para $19,4 \%$.

Como a assimilação das recomendações sobre profilaxia por parte dos médicos não pode ser diretamente medida com este desenho de estudo e podem ter ocorrido variações, dependendo das iniciativas de cada hospital, os resultados deste estudo revelam apenas o impacto potencial destas medidas. De fato, um dos hospitais só teve uma palestra de educação continuada sobre o TEV, enquanto os outros tiveram pelo menos três e um chegou a ter sete palestras no período do estudo. Sendo assim, é pertinente analisar se o tipo de hospital influenciou a utilização de profilaxia. De um modo geral, houve uma tendência a uma melhor adequação do uso de profilaxia farmacológica em hospitais privados, em relação aos públicos e com residência médica, $63,5 \%$ vs. 55,6\%, $p=0,1$. Estas análises, entretanto, não eram os objetivos principais do estudo. Assim, é provável que o pequeno tamanho da amostra não tenha permitido a avaliação de uma diferença realmente significante entre os tipos de hospitais. Por fim, serão necessários novos estudos que possam demonstrar que, quando há vontade das chefias e empenho dos profissionais envolvidos na prevenção do TEV, a avaliação rotineira do risco e o consequente uso adequado da profilaxia são factíveis e podem contribuir de maneira significativa na redução do risco de TEV.

Conflito de interesse: não há 


\section{SUMMARY}

\section{IMPACT OF A PROGRAM FOR VENOUS THROMBOEMBOLISM PROPHYLAXIS}

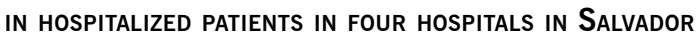

There is a discrepancy between guideline recommendations and practice of venous thromboembolism (VTE) prophylaxis in hospitals worldwide.

OBJECTIVE. To implement a program using a risk-assessment tool (RAT) for VTE and educational lectures based on the Brazilian Guidelines for VTE Prophylaxis for Medical Patients and to evaluate the impact of these tools on adequacy of VTE prophylaxis in 4 hospitals in Salvador, Bahia.

METHODS. We performed two cross-sectional surveys before and after the implementation of the program to compare the proportion of patients at-risk of VTE and the changes in the adequacy of VTE prophylaxis.

RESULTS. We compared the data of 219 medical patients before with 292 patients after the program. The rates of patients with at least one risk factor for VTE and with contraindications (CI) for heparins were similar: 95\% vs. 98\% ( $p=0.13)$, and $42 \%$ vs. 34\% ( $p=0.08$ ), respectively. In both studies, $75 \%$ vs. $82 \%(p=0.06)$ were candidates for prophylaxis, and $44 \% \mathrm{vs}$. $55 \%(p=0.02)$ were candidates for prophylaxis and had no $\mathrm{Cl}$ for heparin. After the program there was an increase in the use of mechanical prophylaxis $0.9 \%$ vs. $4.5 \%(p=0.03)$ and a decrease in pharmacological prophylaxis, $55.3 \%$ vs. $47.9 \%$ $(p=0.04)$. However, there was a significant increase of use of the recommended doses of heparins, 53\% vs. 75 ( $p<0.001)$.

CONCLUSION. There is underutilization of VTE prophylaxis in Brazilian hospitals. Strategies based on passive distribution of RAT and educational lectures were not sufficient to improve the practice of prophylaxis, but improved the adequacy of VTE prophylaxis in hospitalized patients. [Rev Assoc Med Bras 2010; 56(2): 197-203]

KEY WORDS: Venous thromboembolism. Risk factors. Disease prevention. Internal medicine. Practice guidelines as topic. Heparin.

\section{REFERÊNCIAS}

1. Kleber FX, Witt C, Vogel G et al. Randomized comparison of enoxaparin with unfractionated heparin for the prevention of venous thromboembolism in medical patients with heart failure or severe respiratory disease. Am Heart J. 2003;145(4):614-21.

2. Samama MM, Cohen AT, Darmon JY, Desjardins L, Eldor A, Janbon C et al. A comparison of enoxaparin with placebo for the prevention of venous thromboembolism in acutely ill medical patients. Prophylaxis in Medical Patients with Enoxaparin Study Group. N Engl J Med. 1999;341(11):793-800.

3. Alikhan R, Cohen AT, Combe S, Samama MM, Desjardins L, Eldor A, et al. Prevention of venous thromboembolism in medical patients with enoxaparin: a subgroup analysis of the MEDENOX study. Blood Coagul Fibrinolysis, $2003 ; 14(4): 341-6$.
4. Alikhan R, Cohen AT, Combe S, Samama MM, Desjardins L, Eldor A, et al. Risk factors for venous thromboembolism in hospitalized patients with acute medical illness: analysis of the MEDENOX Study. Arch Intern Med. 2004;164(9):963-8.

5. Cohen AT, Davidson BL, Gallus AS, Lassen MR, Prins MH, Tomkowski M, et al. Efficacy and safety of fondaparinux for the prevention of venous thromboembolism in older acute medical patients: randomised placebo controlled trial. BMJ. 2006;332(7537):325-9.

6. Harenberg J, Roebruck P, Heene DL. Subcutaneous low-molecular-weight heparin versus standard heparin and the prevention of thromboembolism in medical inpatients. The Heparin Study in Internal Medicine Group. Haemostasis. 1996;26(3):127-39

7. Leizorovicz A, Cohen AT, Turpie AG, Olsson CG, Vaitkus PT, Goldhaber SZ. Randomized, placebo-controlled trial of dalteparin for the prevention of venous thromboembolism in acutely ill medical patients. Circulation. 2004;110(7):874-9.

8. Cohen AT, Tapson VF, Bergmann JF, Goldhaber SZ, Kakkar AK, Deslandes B, et al. Venous thromboembolism risk and prophylaxis in the acute hospital care setting (ENDORSE study): a multinational cross-sectional study. Lancet. 2008;371(9610):387-94.

9. Tapson VF, Decousus H, Pini M, Chong BH, Froehlich JB, Monreal M, et al. Venous thromboembolism prophylaxis in acutely ill hospitalized medical patients: findings from the International Medical Prevention Registry on Venous Thromboembolism. Chest. 2007;132(3):936-45.

10. Rashid ST, Thursz MR, Razvi NA, Voller R, Orchard T, ShlebakAA. Venous thromboprophylaxis in UK medical inpatients. J R Soc Med. 2005;98(11):507-12.

11. Arnold DM, Kahn SR, Shrier I. Missed opportunities for prevention of venous thromboembolism: an evaluation of the use of thromboprophylaxis guidelines. Chest. 2001;120(6):1964-71.

12. Rocha AT, Braga P, Ritt G, Lopes AA. [Inadequacy of thromboprophylaxis in hospitalized medical patients]. Rev Assoc Med Bras. 2006;52(6):441-6.

13. Menna Barreto SS, Faccin CS, Silva PM, Cneteno LP, Gazzana MB. Estratificação de risco e profilaxia para tromboembolia venosa em pacientes internados em hospital geral universitário. J Pneumol.1998;24(5):298-302.

14. Menna Barreto SS, Silva PM, Faccin CS, Theil AL, Nunes AH, Pinheiro CTS. Profilaxia para tromboembolia venosa em uma unidade de tratamento intensivo. J Pneumol. 1999;26(1):15-9.

15. Caiafa JS, Moura LK, Raymundo S. Managing venous thromboembolism in Latin American patients: emerging results from the Brazilian Registry. Semin Thromb Hemost. 2002;28(Suppl 3):47-50.

16. Rocha AT, Paiva EF, Lichtenstein A, Milani JrR, Cavalheiro-Filho C, Maffei FH, et al. Diretriz Brasileira para Profilaxia de Tromboembolismo Venoso em Pacientes Clínicos. In: Projeto diretrizes. Associação Médica Brasileira, Conselho Federal de Medicina. São Paulo:Associação Médica Brasileira; 2006.

17. Kucher N, Koo S, Quiroz R, Cooper JM, Paterno MD, Soukonnikov B, et al. Electronic alerts to prevent venous thromboembolism among hospitalized patients. N Engl J Med. 2005;352(10):969-77.

18. Sad EF, Santos RS, Braga AC. Improvement of venous thromboembolism prophylaxis in hospitalized patients after an educational program. 2008.

19. Rocha AT, Paiva EF, Lichtenstein A, Milani Jr R, Cavalheiro CF, Maffei FH. Riskassessment algorithm and recommendations for venous thromboembolism prophylaxis in medical patients. Vasc Health Risk Manag. 2007;3(4):533-53.

20. Wein L, Wein S, Haas SJ, Shaw J, Krum H. Pharmacological venous thromboembolism prophylaxis in hospitalized medical patients: a meta-analysis of randomized controlled trials. Arch Intern Med. 2007;167(14):1476-86.

Artigo recebido: 14/04/09 Aceito para publicação: 25/10/09 